\title{
Moderne Gesellschaft, nichtaffirmative Erziehung und das Problem der Kontroversität
}

Die Frage, wie Erziehung beschrieben werden muss, damit die Beschreibung als eine sinnvolle Antwort auf die Situation gewertet werden kann, in der Menschen in modernen Gesellschaften aufwachsen, ist ein Dauerproblem pädagogischer Theoriebildung. In der europäischen und insbesondere deutschsprachigen Tradition sind von verschiedener Seite Beschreibungen von Erziehung angefertigt worden, die eine gemeinsame Grundausrichtung erkennen lassen. Man kann diese im Detail durchaus voneinander abweichenden Beschreibungen als Beiträge zu einem gemeinsamen ,Projekt' auffassen, nämlich eine Theorie nichtaffirmativer Erziehung zu entwerfen. ${ }^{1}$ Gemeint ist damit ein Theorieansatz, in dem versucht wird, die Eigenlogik moderner Erziehung in den Blick zu rücken, wie sie u.a. von Jean-Jacques Rousseau, Johann Gottlieb Fichte, Friedrich Schleiermacher oder Johann Friedrich Herbart unter verschiedenen Schwerpunktsetzungen beschrieben worden ist. Die Theorie nichtaffirmativer Erziehung lässt sich als eine „present-day reconstruction of this modern tradition"2 verstehen. Erziehung wird in diesem Zusammenhang als eine Form des Miteinanderumgehens begriffen, in der Heranwachsende so in die Welt eingeführt werden, dass diese eine vielseitig dimensionierte Urteils- und Partizipationsfähigkeit entwickeln können, die es ihnen ermöglicht, ihr Leben im Kontext einer modernen demokratischen Gesellschaft selbstbestimmt zu führen.

Die Entwicklung dieser Theorie ist vor dem Hintergrund der grundlegenden gesellschaftlichen Transformationsprozesse zu sehen, die sich in Kontinentaleuropa insbesondere in dem Zeitraum zwischen 1700 und 1850 vollzogen haben. ${ }^{3}$ In dieser Zeit haben Gesellschaften damit begonnen, modern zu werden, wobei Modernisierung hier einen gesellschaftlichen Ausdifferenzierungsprozess bezeichnet. Heute beschreiben sich moderne Gesellschaften als komplexe Gesellschaften, die in unterschiedliche Kontexte ausdifferenziert sind, die jeweils nach eigenen Logiken funktionieren. Jeder dieser Kontexte - Wissenschaft, Politik, Religion, Kunst, Wirtschaft, Recht oder Moral - offeriert eine spezifische Perspektive auf Sachverhalte, ohne dass die Möglichkeit besteht, auf eine allgemein akzeptierte Regel zurückzugreifen, die es erlauben würde, dass Wechselspiel der Perspektiven in die allein ,richtige' Ordnung zu überführen. ${ }^{4}$

Es verwundert vor diesem Hintergrund nicht, dass im Zuge dieser gesellschaftlichen Transformationsprozesse auch neue Beschreibungen von Erziehung angefertigt worden sind - Beschreibungen, in denen erste Versuche beobachtet werden können, Erziehung relational zu der sich abzeichnenden Ausdifferenzierung der Gesellschaft als nichtaffirmative Erziehung 
neu zu bestimmen. So gesehen wäre zu erwarten, dass ein solcher Ansatz sozusagen von Grund auf ,sensibel' dafür ist, dass das Aufwachsen in modernen Gesellschaften unter den Bedingungen einer Vielheit von Perspektiven ihren Ort hat Perspektiven, die nicht notwendigerweise miteinander harmonieren, sondern sich auch widersprechen können. Tatsächlich findet sich bei Herbart die Idee, dass Unterricht ein ,vielseitiges' Weltverstehen ermöglichen sollte, und Schleiermacher spricht der Erziehung im Allgemeinen die Aufgabe zu, den Einzelnen ${ }^{5}$ in den ,Streit einzuführen, der in der Öffentlichkeit moderner Gesellschaften ausgemacht werden kann. ${ }^{6}$

Vor diesem Hintergrund verwundert es umso mehr, dass die Frage, wie mit dem Umstand, dass viele Sachverhalte in modernen Gesellschaften kontrovers diskutiert werden, pädagogisch umzugehen ist, im Kontext einer Theorie nichtaffirmativer Erziehung - insgesamt betrachtet - bislang nur bedingt Aufmerksamkeit erfahren hat. Die Beantwortung dieser Frage begnügt sich in der Regel mit einem Hinweis auf die Maßgabe, dass diejenigen Sachverhalte, die in einer Gesellschaft kontrovers diskutiert werden, auch in der Erziehung - zumindest im Kontext öffentlicher Erziehung - als kontrovers thematisiert werden müssen. Diese Antwort ist nicht ohne Probleme, weshalb es auch nicht weiter verwundert, dass die besagte Antwort mit Alternativen konfrontiert werden kann, wie neuere Debatten innerhalb der internationalen Philosophy of Education zeigen. ${ }^{7}$ In der Theorie nichtaffirmativer Erziehung ist von diesen Debatten bislang keine Notiz genommen worden. Umgekehrt liegt in Bezug auf die besagte Kontroverse über das Kontroversitätsgebot öffentlicher Erziehung bislang keine Stellungnahme vor, die ausgehend von den Grundannahmen und -begriffen einer Theorie nichtaffirmativer Erziehung entwickelt worden wäre.
Welche Sachverhalte sollen im Kontext öffentlicher Erziehung als kontrovers thematisiert werden? Das ist die Problemstellung, die ich in diesem Beitrag in Bezug auf die Theorie nichtaffirmativer Erziehung bearbeiten und einer möglichen Lösung zuführen möchte. Hierdurch soll insbesondere eine Leerstelle gefüllt werden, die in der Theorie nichtaffirmativer Erziehung bislang nicht die gebührende Aufmerksamkeit erfahren hat. Daneben ist mit den folgenden Überlegungen aber auch die Hoffnung verknüpft, einen Beitrag zur aktuellen Debatte um einen angemessenen Umgang mit kontroversen Sachverhalten zu leisten, indem eine pädagogische Perspektive in diese Debatte eingebracht wird. Dahinter steht die Vermutung, dass die Kontroverse, die in der Philosophy of Education seit der bahnbrechenden Arbeit What should we teach as controversial? von Michael Hand ${ }^{8}$ neu entfacht worden ist, eine spezifische Perspektive widerspiegelt, wie sie für grundlagenreflexive Theoriearbeit im anglo-amerikanischen Sprachraum insgesamt typisch zu sein scheint. Erziehung wird hier - primär jedenfalls - im Lichte spezifischer philosophischer Prämissen thematisiert, nicht aber so, dass von der Eigenlogik moderner Erziehung her argumentiert wird - und damit im Lichte einer Perspektive, wie sie insbesondere im deutschen Sprachraum entwickelt worden ist. In diesem Sinne möchte ich zumindest auch untersuchen, ob und, falls ja, wie das Problem der Kontroversität formuliert und bearbeitet werden kann, wenn nicht „philosophical questions", sondern "educational questions about education" als Ausgangspunkt der Theoriebildung fungieren. $^{9}$ Allerdings - dies sei bereits vorab einschränkend gesagt - werde ich angesichts des zur Verfügung stehenden Platzes keine ausführliche Diskussion der verschiedenen Positionen vornehmen, die in der Debatte 
um das Kontroversitätsgebot öffentlicher Erziehung eingenommen werden. Der Schwerpunkt des Beitrags liegt darauf, die Theorie nichtaffirmativer Erziehung weiterzuentwickeln, indem eine Stellungnahme zum Problem der Kontroversität entworfen wird. In diesem Sinne sind die folgenden Überlegungen auch nicht auf eine Erörterung spezifischer Sachverhalte gerichtet, die (aktuell) in der Öffentlichkeit kontrovers diskutiert werden (Klimawandel, Migration, Pandemiebekämpfung, Digitalisierung etc.). Es geht mir vielmehr darum, einen theoretischen Rahmen zu entwickeln, der erziehungs- und bildungstheoretisch ausgewiesen ist, und der dazu beitragen soll, eine Beurteilung entsprechender Sachverhalte aus pädagogischer Perspektive vorzunehmen.

Der Beitrag gliedert sich wie folgt: Zunächst rekonstruiere ich Grundannahmen und -begriffe einer Theorie nichtaffirmativer Erziehung (1.-3.). Darauf aufbauend arbeite ich heraus, welche Antwort in diesem Zusammenhang bislang auf die Frage gegeben worden ist, wie aus pädagogischer Warte mit kontroversen Sachverhalten umgegangen werden sollte (4./5.). Schließlich entwickle ich einen alternativen Lösungsvorschlag, der eine spezifische Umgrenzung derjenigen Sachverhalte vorsieht, die im Kontext öffentlicher Erziehung als kontrovers thematisiert werden sollten - eine Umgrenzung, die in Bezug auf den übergeordneten Anspruch nichtaffirmativer Erziehung, Bildung zu ermöglichen, gerechtfertigt werden kann (6.-10.).

\section{Affirmative vs. nichtaffirmative Erziehung}

Menschen - dies ist die Grundprämisse der folgenden Überlegungen - sind imperfekte, d.h. unfertige Wesen, die in der Notwendigkeit existieren, praktisch tätig zu werden. Indem Menschen handeln, bringen sie Kultur hervor, wobei Kultur hier in einem weiten Sinne verstanden wird. Diese geht nicht in Literatur, Kunst, Philosophie oder Religion auf, sondern umfasst u.a. auch Naturwissenschaft, Technik, Politik oder Wirtschaft. Indem Menschen kulturschaffend tätig sind, wenden sie ihre Imperfektheit, ohne sie aufheben zu können. ${ }^{10}$

Kultur tradiert sich nicht von selbst. Das bedeutet, dass Menschen kulturelle Sachverhalte (ein bestimmtes Wissen, ein bestimmtes Können, bestimmte $\mathrm{Hal}$ tungen etc.) - sollen diese über die Mortalitätsschwelle hinübergerettet werden - tradieren müssen. Umgekehrt müssen Menschen, die in eine Kultur hineingeboren werden, in diese eingeführt werden. Kultur tradiert sich nicht nur nicht von selbst, sie versteht sich in vielen Fällen auch nicht von selbst. Kurzum: Kultur muss vermittelt und angeeignet werden, damit eine bereits begonnene Geschichte fortgesetzt werden kann. ${ }^{11}$ Es ist dieser Zusammenhang von Vermittlungs- und Aneignungsaktivitäten, den ich mit dem Begriff der Erziehung bezeichne. Beide Aktivitäten sind auf ein Drittes bezogen, nämlich auf den kulturellen Sachverhalt, der vermittelt und angeeignet werden soll. Wenn wir von Erziehung sprechen, so gilt es neben dem Subjekt der Vermittlung - dem Erzieher - und dem Subjekt der Aneignung - dem Edukanden - noch einen ,dritten Faktor' zu unterscheiden, nämlich den kulturellen Sachverhalt, der über die Mortalitätsschwelle hinübergerettet bzw. in den ein ,Neuankömmling eingeführt werden soll. Erziehung reagiert in diesem Sinne auf das Problem der Mortalität und auf das Problem der Natalität gleichermaßen. ${ }^{12}$ So gesehen erfült Erziehung nicht nur die Funktion, in kulturelle Sachverhalte einzuführen, die als Antworten auf bestimmte Facetten menschlicher Imperfektheit begriffen werden können. Erziehung gründet selbst in einer 
spezifischen Facette menschlicher Imperfektheit, nämlich dem Umstand, dass kulturelle Sachverhalte sich nicht von selbst tradieren, sondern vermittelt und von jeder Generation neu angeeignet werden müssen. In diesem Sinne kann Erziehung als konstitutiv für menschliches Leben und Zusammenleben angesehen werden.

Es dürfte unmittelbar einleuchten, dass Erziehung nicht notwendigerweise als nichtaffirmativ begriffen werden muss. Vielmehr handelt es sich hierbei um eine normative Ausrichtung im pädagogischen Denken, die irreduzibel mit einem gesellschaftlichen Transformationsprozess verknüpft ist, der sich in Zentraleuropa vor allem im Zeitraum zwischen 1700 und 1850 vollzogen hat, und in dem die Menschen damit begonnen haben, sich selbst als Freie und Gleiche zu verstehen, die in Solidarität miteinander verbunden sind. Die Idee einer wechselseitigen Achtung der Menschen als Menschen wird in diesem Prozess, den wir heute als Modernisierung westlicher Gesellschaften beschreiben, zu einem zentralen Orientierungsgesichtspunkt - auch im pädagogischen Denken. Dietrich Benner weist in diesem Sinne explizit darauf hin, dass "die im kategorischen Imperativ formulierte Aufgabe", den Einzelnen niemals nur als Mittel zum Zweck, sondern stets zugleich als Selbstzweck zu behandeln für die "Pädagogik" das „Problem" aufgeworfen hat (und bis heute aufwirft), "wie die Aufgaben und Möglichkeiten einer Erziehung zu bestimmen sind", welche die Entwicklung von Urteils- und Handlungsfähigkeit zu ermöglichen sucht, "Ohne diese vorweg zu normieren". ${ }^{13}$

Systematisch betrachtet kann Erziehung spätestens seit dieser Zeit in einem affirmativen und in einem nichtaffirmativen Sinne begriffen werden ${ }^{14}$, wobei affirmative Erziehung selbst noch einmal in unterschiedlichen Varianten in Erscheinung tritt, nämlich als affirmativ-bewahrende und als affirmativ-emanzipative Erziehung. ${ }^{15}$ Das
Gemeinsame beider Varianten besteht darin, dass der Erziehung jeweils die Aufgabe zugeschrieben wird, Heranwachsende auf eine bestimmte Ordnung hin festzulegen - sei es eine gegebene, sei es eine stellvertretend antizipierte Ordnung. Dies ist deshalb wichtig zu betonen, weil man leicht dem Missverständnis aufsitzen kann, nichtaffirmative Erziehung sei auf eine Positionierung der Heranwachsenden gegen eine tradierte Ordnung gerichtet. Demgegenüber gilt es festzuhalten, dass auch eine Erziehung, die auf den Widerstand gegenüber einer gesellschaftlichen Ordnung hin finalisiert ist, letztlich nichts anderes als eine spezifische Spielart affirmativer Erziehung bedeutet. Die Frage, $o b$ Widerstand geleistet werden sollte oder nicht, gilt hier nämlich bereits vor aller Erziehung als entschieden.

Im Unterschied zu einer Theorie affirmativer Erziehung, in der die Erziehung auf ein Mittel zur Durchsetzung gesellschaftlicher Erwartungen an die nachwachsende Generation reduziert wird, rückt eine Theorie nichtaffirmativer Erziehung die Erziehung als eine tradierende und transformierende Praxis in den Blick. Die Frage, was im Leben und Zusammenleben vorzuziehen bzw. zurückzustellen ist, wird im Kontext einer Theorie nichtaffirmativer Erziehung als eine Frage begriffen, die gerade nicht schon vor aller Erziehung ihre Beantwortung erfahren hat, so dass Erziehung nur mehr bereits gefundene Antworten zu tradieren hätte. Nichtaffirmative Erziehung tradiert die besagte Frage vielmehr als Frage, d.h. sie ist darauf gerichtet, die Heranwachsenden in die Suche nach Orientierung eines gelingenden Lebens und Zusammenlebens selbst hineinzuziehen. Dies schließt freilich mit ein, die Heranwachsenden zu einer Reflexion auf die Antworten zu veranlassen, die Menschen in der Geschichte auf ihrer Suche nach Orientierung bereits gefunden haben. Diese Antworten geben allerdings 
nicht das Maß vor, an dem Erziehung auszurichten wäre, sondern avancieren in der Erziehung selbst zum Gegenstand der Auseinandersetzung - was freilich die Möglichkeit impliziert, dass die Heranwachsenden die Tradition auf eigene Art und Weise transformieren.

Eine Theorie nichtaffirmativer Erziehung zu entwickeln, lässt sich vor diesem Hintergrund als der Versuch interpretieren, eine Erziehung zu beschreiben, in der das Denken, Urteilen und Handeln des Edukanden nicht normiert wird. Gerade darin erweist sich die Theorie selbst als nicht neutral. Diese bringt vielmehr eine normative Position zum Ausdruck, die ausdrücklich gegen Versuche gerichtet ist, Erziehung für eine Durchsetzung gesellschaftlicher Zwecke zu instrumentalisieren. Nichtaffirmative Erziehung nimmt ihren Ausgang zwar von gesellschaftlichen Erwartungen, die an die Heranwachsenden adressiert werden, affirmiert diese jedoch nicht, sondern behandelt sie als Bestimmungen, die Menschen hervorgebracht haben, und die gerade deshalb, weil Menschen imperfekte Lebewesen sind, zukünftig erneut zu Gegenständen und Ausgangspunkten der Wechselwirkung von Mensch und Welt werden können. Dies ist, mit Michael UIjens gesprochen, der spezifische "moral imperative inherent in this theory "16, wie er traditionell im Begriff der Bildung zum Ausdruck gebracht wird, nämlich Heranwachsenden in der Erziehung immer auch die Möglichkeit zu eröffnen, sich zu ,externen' Ansprüchen in ein Verhältnis zu setzen, um so „experimentelle, selbstgewählte Bestimmungen" ${ }^{17}$ hervorzubringen.

\section{Die Eigenlogik moderner Erziehung}

Erziehung an die Aufgabe einer Freisetzung des Einzelnen für ein Leben in Selbstbestimmung zu knüpfen, darf nicht relativistisch missverstanden werden. Erziehung ist, wie Marian Heitger formuliert, gerade „um der Bildung des jungen Menschen willen“ dazu verpflichtet, „den Anspruch von Niveau und Wertigkeit der Tradition gegenüber dem jungen Menschen zur Geltung zu bringen“. Dies ist - wie man zunächst vielleicht meinen könnte - kein „Widerspruch zur pädagogischen Aufgabe", dem Heranwachsenden die „Freiheit der Stellungnahme den Gegebenheiten gegenüber" zu eröffnen. ${ }^{18}$ Vielmehr handelt es sich hierbei um „ihre konkrete Möglichkeit"19, womit klar sein dürfte, dass das Sich-Verhalten, zu dem nichtaffirmative Erziehung auffordert, nicht mit dem Kundtun (vermeintlich) eigener Meinungen verwechselt darf, sondern vielmehr ein ,Durcharbeiten“ von Differenzerfahrungen impliziert, die sich in der Konfrontation des Heranwachsenden mit einer widerständigen Welt einstellen. Allerdings geht eine Erziehung als Ermöglichung von Bildung auch nicht darin auf, den Einzelnen mit einer widerständigen Welt zu konfrontieren, und diesem dabei zu helfen, Differenzerfahrungen durchzuarbeiten. Bildung schließt vielmehr mit ein, dass der Einzelne „im Blick auf seine eigene Kultur sich als urteilendes und handelndes Subjekt ins Spiel bringt"20. Nichtaffirmative Erziehung bedeutet vor diesem Hintergrund auch und vor allem, dem Einzelnen die Möglichkeit zu eröffnen, sich zur Welt - und zwar in ihrer Widerständigkeit - zu verhalten sowie die eigenen Positionsbestimmungen selbst wiederum potentiellen Differenzerfahrungen auszusetzen (z.B. in der Konfrontation mit Alternativen), um so zu Positionen zu finden, die mit der Widerständigkeit der Welt gleichsam ,abgestimmt' sind. ${ }^{21}$

Eine solche Aufgabenbestimmung von Erziehung korreliert mit einer spezifischen Form des Umgangs des Erziehers mit dem Edukanden. Der Begriff, mit dem diese Form im Kontext einer 
Theorie nichtaffirmativer Erziehung bezeichnet wird, lautet Aufforderung zur Selbsttätigkeit. Allerdings ist es in diesem Zusammenhang nötig, Unterscheidungen zu beachten, um nicht vorschnell möglichen Missverständnissen aufzusitzen. Zunächst ist es hilfreich, zwischen einem ,basalen' und einem ,anspruchsvollen“ Verständnis von Aufforderung zur Selbsttätigkeit zu unterscheiden.

Erziehung in einem basalen Sinne als Aufforderung zur Selbsttätigkeit zu bestimmen, trägt dem Umstand Rechnung, dass der Einzelne in seinem Lernen prinzipiell unvertretbar ist. Zugespitzt formuliert: Lernen kann jeder nur für sich selbst. Das bedeutet, dass Lernen nicht im strengen Sinne bewirkt, sondern immer nur ermöglicht werden kann. Ich habe diesen Umstand, der letztlich nicht nur für eine nichtaffirmative, sondern auch für Spielarten einer affirmativen Erziehung gilt, dadurch zu berücksichtigen versucht, dass ich Erziehung als einen Zusammenhang zweier Aktivitäten beschrieben habe, die nicht auseinander abgeleitet, durcheinander ersetzt oder aufeinander reduziert werden können: Vermittlung und Aneignung. ${ }^{22}$

Im Unterschied hierzu bringt ein anspruchsvoller Begriff von Aufforderung zur Selbsttätigkeit Erziehung als eine Form des Miteinanderumgehens in den Blick, in der Heranwachsende mit einer widerständigen Welt konfrontiert werden, die Ansprüche an den Einzelnen richtet, die dessen Selbstbezogenheit gleichsam durchkreuzen. Zur Selbsttätigkeit aufzufordern, bedeutet darüber hinaus, dem Einzelnen dabei zu helfen, Widerstandserfahrungen durchzuarbeiten - dies aber so, dass dem Einzelnen die Möglichkeit eröffnet wird, sich zu den jeweiligen Ansprüchen noch einmal in ein Verhältnis zu setzen. Erziehung in diesem Sinne als Aufforderung zur Selbsttätigkeit zu begreifen, ist kennzeichnend für eine Theorie nichtaffirmativer Erziehung, nicht aber für
Beschreibungen von Erziehung, in denen Erziehung als affirmativ bestimmt wird.

Gert Biesta hat an dieser Stelle eine weitere Differenz vorgeschlagen, die mir hilfreich zu sein scheint, um einem anspruchsvollen Begriff von Aufforderung zur Selbsttätigkeit noch mehr Kontur zu verleihen. Ich habe oben bereits eine mögliche Fehlinterpretation dieses Begriffs angedeutet, als ich darauf hingewiesen habe, dass nichtaffirmative Erziehung nicht damit verwechselt werden sollte, Heranwachsende zur Artikulation, eigener' Meinungen und in diesem Sinne zur ,Selbsttätigkeit' aufzufordern. Um ein solches Missverständnis zu vermeiden, votiert Biesta dafür, zwischen der Aufforderung ,Be yourself!' und der Aufforderung ,Be a self!' zu unterscheiden, wobei er allein letzterer Aufforderung einen pädagogischen Wert beimisst. ${ }^{23}$ Im Kontext einer Theorie nichtaffirmativer Erziehung wird Aufforderung zur Selbsttätigkeit - dies sollte deutlich geworden sein - im Sinne eines, Be a self!' verstanden, d.h. gerade nicht als Aufforderung, zu zeigen, wer man ist, sondern als Aufforderung, zu zeigen, wie man sich im Durchgang durch eine Differenzerfahrung zu etwas positioniert. Während im ersten Fall nach der Identität einer Person gefragt wird, die sich im Zuge des Aufwachsens entwickelt hat, geht es im zweiten Fall darum, den Heranwachsenden als Subjekt zu adressieren.

Eine Theorie, in der Erziehung in einem anspruchsvollen Sinne als Aufforderung zur Selbsttätigkeit begriffen wird, operiert nolens volens mit einer spezifischen anthropologischen Prämisse, nämlich dass der Einzelne potentiell dazu fähig ist, Differenzerfahrungen durch eigene Entwürfe zu wenden, und in diesem Sinne ein Anderer zu werden, für den sich auch die Welt zukünftig anders darstellt. In der Theorie nichtaffirmativer Erziehung steht für diese Annahme der Begriff der Bildsamkeit. ${ }^{24}$ Ohne die Voraussetzung, dass der Mensch 
bildsam, d.h. von Natur aus unbestimmt und zugleich dazu fähig sei, über Aktivitäten des Selber-Denkens, Selber-Urteilens und Selber-Handelns neue Fähigkeiten zu entwickeln, wäre es sinnlos, Erziehung überhaupt in einem nichtaffirmativen Sinne zu begreifen. Umgekehrt wird der Einzelne immer schon als bildsam vorausgesetzt, wenn dieser als Subjekt adressiert wird.

Den Einzelnen als bildsam zu begreifen, bedeutet in diesem Zusammenhang nicht nur, diesen als ansprechbar für Erziehung aufzufassen. Es bedeutet aber auch nicht nur, den Einzelnen als jemanden zu verstehen, der im Verhältnis zu einer widerständigen Welt eigene Positionen entwickeln kann. Bildsamkeit meint darüber hinaus, den Einzelnen als jemanden zu begreifen, der sich selbst - potentiell jedenfalls - als Subjekt verstehen kann. Nichtaffirmative Erziehung ist auch und vor allem darauf gerichtet, diese Facette von Bildsamkeit anzusprechen und dem Einzelnen die Erfahrung zu ermöglichen, dass er sich zur Welt in ein Verhältnis setzen kann. Mit anderen Worten: Den Einzelnen zur Selbsttätigkeit aufzufordern und ihn z.B. in Wahlsituationen hineinzuziehen, soll diesen nicht nur dazu veranlassen, eine Wahl zu treffen, sondern ihm auch die Möglichkeit eröffnen, sich selbst als Wählenden zu begreifen. Dies zu betonen, ist deshalb wichtig, weil die Erfahrung, Subjekt sein zu können, eine Voraussetzung dafür sein dürfte, dass der Einzelne von sich aus damit beginnt, sich zur Welt in ein Verhältnis zu setzen, und Erziehung damit fortschreitend überflüssig werden kann.

\section{Gesellschaftliche Verflechtungen}

Die Prinzipien der Selbstbestimmung, der Bildsamkeit und der Aufforderung zur Selbsttätigkeit rücken in ihrem Zusammenhang die Eigenstruktur moderner
Erziehung in den Blick ${ }^{25}$, wobei diese im Detail durchaus auf unterschiedliche Art und Weise ausbuchstabiert werden kann. Entscheidend ist an dieser Stelle aber etwas anderes, nämlich dass es sich hierbei um eine "programmatische Struktur" 26 handelt. Das bedeutet, dass nicht davon ausgegangen werden kann, dass eine an den Prinzipien der Bildsamkeit, der Selbstbestimmung und der Aufforderung zur Selbsttätigkeit orientierte Erziehung in modernen demokratischen Gesellschaften bereits immer und überall realisiert wäre. Die Beschreibung der Eigenstruktur moderner Erziehung, wie sie in der Theorie nichtaffirmativer Erziehung angefertigt wird, besitzt in diesem Sinne ein gesellschaftskritisches Potential. Dieses speist sich jedoch nicht aus einer stellvertretend antizipierten gesellschaftlichen Ordnung, sondern aus der Frage, ob und, falls ja, inwieweit nichtaffirmative Erziehung unter den gegebenen gesellschaftlichen Bedingungen möglich ist.

Was damit gemeint ist, lässt sich präziser fassen, wenn wir die konstitutiven Prinzipien moderner Erziehung (Selbstbestimmung, Bildsamkeit und Aufforderung zur Selbsttätigkeit) um zwei regulative Prinzipien ergänzen. Diese bringen nicht die Eigenlogik moderner Erziehung, sondern vielmehr das Verhältnis zwischen einer nichtaffirmativen Erziehung und anderen gesellschaftlichen Bereichen auf Begriffe. Dass eine Theorie nichtaffirmativer Erziehung dieses Verhältnis mitreflektieren muss, beruht auf der Annahme, dass Erziehung in gesellschaftliche Zusammenhänge eingebunden ist, wobei andere gesellschaftliche Bereiche nach alternativen Logiken funktionieren. Aus diesem Grund kann es nicht als selbstverständlich angesehen werden, dass die Erziehung entsprechend ihrer modernen Eigenlogik operiert bzw. operieren kann. Die Möglichkeit einer nichtaffirmativen Erziehung ist vielmehr davon abhängig, ob und, falls ja, 
inwieweit in einer Gesellschaft das Prinzip der Transformation gesellschaftlicher in pädagogisch legitime Ansprüche sowie das Prinzip der Nichthierarchizität Anerkennung finden, wobei sich insbesondere letzteres für die Formulierung und Bearbeitung des Problems der Kontroversität als bedeutsam erweisen wird.

Das Transformationsprinzip besagt u.a., dass gesellschaftliche Erwartungen an die nachwachsende Generation eine Umformung durchlaufen müssen, damit Heranwachsende überhaupt in ihrer Bildsamkeit anerkannt, zur Selbsttätigkeit aufgefordert und für ein Leben in Selbstbestimmung freigesetzt werden können. ${ }^{27}$ Gesellschaftliche Ansprüche müssen in Sachverhalte transformiert werden, die Heranwachsende verstehen und zu denen sie sich in ein Verhältnis setzen können. Dies schließt u.a. mit ein, dass nicht schon vorab normiert sein darf, wie der Einzelne sich zu dem jeweiligen Sachverhalt verhält. Das bedeutet, dass gesellschaftliche Ansprüche im Kontext einer nichtaffirmativen Erziehung ihre Geltung in einem bestimmten Sinne einbüßen müssen, sollen Heranwachsende überhaupt als Subjekte adressiert werden können. Was dies konkret bedeuten kann, lässt sich z.B. anhand des Religionsunterrichts illustrieren. In einem Religionsunterricht, der auf die Ermöglichung von Bildung gerichtet ist, wird die in einer Religion verkündete Botschaft nicht einfach proklamiert, sondern thematisiert, d.h. interpretiert, befragt und - unter Umständen - problematisiert. ${ }^{28}$ Aspekte einer Religion, die innerhalb der jeweiligen Religion in ihrer Geltung außer Frage stehen, müssen diese Geltung im Religionsunterricht verlieren, wenn dieser auf eigene Urteilsbildung und die Entwicklung einer entsprechenden Partizipationsfähigkeit ausgerichtet sein soll.

Die Möglichkeit einer pädagogischen Transformation gesellschaftlicher Ansprüche ist selbst wiederum davon abhängig, dass andere gesellschaftliche Bereiche davon absehen, die Erziehung in ihren Dienst zu nehmen. Dies gilt analog für sämtliche gesellschaftlichen Bereiche. Sollen diese jeweils entsprechend ihrer Eigenlogik operieren können, so kommt es darauf an, dass die verschiedenen Bereiche grundsätzlich davon absehen, einem bestimmten Bereich einen Primat gegenüber den jeweils anderen Bereichen zuzusprechen. Diese Maßgabe bringt das Prinzip der Nichthierachizität auf einen Begriff. ${ }^{29}$

Gerade was das Verhältnis zwischen der Erziehung und den jeweils anderen gesellschaftlichen Bereichen betrifft, mag es zunächst als befremdlich erscheinen, diesen keine Vorrangstellung gegenüber der Erziehung zuzusprechen. Immerhin handelt es sich um eine eingewöhnte Beschreibung von Erziehung, dass diese im Dienste anderer Bereiche stehe bzw. zu stehen habe. Die pädagogische Frage wird dann darauf reduziert, zu klären, wie die jeweiligen gesellschaftlichen Ansprüche so effizient wie möglich realisiert werden können. ${ }^{30}$

Welches Argument lässt sich anführen, um die Idee zu verteidigen, dass der für nichtaffirmative Erziehung maßgebliche Anspruch, den Einzelnen für ein Leben in Selbstbestimmung freizusetzen, von Seiten der jeweils anderen gesellschaftlichen Bereiche akzeptiert werden sollte? Eine mögliche Antwort auf diese Frage könnte wie folgt lauten: Moderne demokratische Gesellschaften sind u.a. dadurch gekennzeichnet, dass Menschen die Möglichkeit haben, ihr Leben selbstbestimmt zu führen, solange sie auch anderen Menschen die Möglichkeit geben bzw. allererst eröffnen, ihr Leben entsprechend zu führen. Dabei wird vorausgesetzt, dass Menschen ihr Leben selbstbestimmt führen können, was - pädagogisch betrachtet - nicht als selbstverständlich angesehen werden kann. Menschen müssen die Fähigkeit, ihr 
Leben im Kontext der verschiedenen gesellschaftlichen Bereiche selbstbestimmt zu führen, erst entwickeln, und sind hierbei u.a. auf eine Erziehung angewiesen, die Heranwachsende als Subjekte adressiert. Eigene Positionen z.B. in Fragen der Berufswahl, aber auch in moralischen, politischen oder religiösen Fragen zu entwickeln, die die Widerständigkeit der Welt berücksichtigen, ist anspruchsvoll, und es ist nicht zu sehen, wie die Entwicklung entsprechender Positionen ohne Erziehung gelingen könnte. Hieraus lässt sich schlussfolgern, dass die Eigenlogik moderner Erziehung Berücksichtigung finden muss, wenn an der ldee festgehalten werden soll, dass Menschen in modernen demokratischen Gesellschaften einen Anspruch darauf haben, ein selbstbestimmtes Leben zu führen. Zugespitzt formuliert: Die Idee der selbstbestimmten Lebensführung geht mit einer spezifischen Logik von Erziehung einher, und diese Logik muss Berücksichtigung finden, sofern überhaupt an der Idee der selbstbestimmten Lebensführung festgehalten werden soll. ${ }^{31}$

\section{Das Kontroversitätsgebot}

In den vorangegangen Abschnitten habe ich Grundannahmen und -begriffe einer Theorie nichtaffirmativer Erziehung erläutert. Im Folgenden möchte ich nun der Frage nachgehen, wie innerhalb des damit gesteckten Rahmens mit der Tatsache umgegangen wird, dass in modernen demokratischen Gesellschaften bestimmte Sachverhalte Gegenstände öffentlicher Kontroversen sind. Dass sich diese Frage gerade im Rahmen einer Theorie nichtaffirmativer Erziehung stellt, zumindest aber stellen müsste, liegt auf der Hand. In dieser Theorie wird nämlich davon ausgegangen, dass in modernen demokratischen Gesellschaften Bereiche unterschieden werden können, die jeweils nach unterschiedlichen
Logiken ,funktionieren'. Darüber hinaus dürfte klar sein, dass es nicht nur zwischen den verschiedenen gesellschaftlichen Bereichen, sondern auch innerhalb dieser Bereiche zu Kontroversen über bestimmte Sachverhalte kommen kann. Nicht nur zwischen Moral, Politik, Religion oder Wissenschaft können in der Öffentlichkeit Kontroversen beobachtet werden, sondern eben auch zwischen verschiedenen moralischen, politischen, religiösen oder wissenschaftlichen Positionen. Vor diesem Hintergrund drängt sich die Frage geradezu auf, wie aus pädagogischer Warte, d.h. unter Berücksichtigung der Eigenlogik moderner Erziehung auf den Umstand, dass bestimmte Sachverhalte in modernen demokratischen Gesellschaften öffentlich kontrovers diskutiert werden, reagiert werden sollte. Wie ich eingangs bereits angedeutet habe, ist es vor diesem Hintergrund mehr als irritierend, dass in der Theorie nichtaffirmativer Erziehung das Problem der Kontroversität bislang kaum behandelt worden ist - schon gar nicht unter Berücksichtigung aktueller Theoriedebatten.

In der Theorie nichtaffirmativer Erziehung wird stattdessen auf den so genannten ,Beutelsbacher Konsens' rekurriert, um zu verdeutlichen, was es bedeutet, kontrovers diskutierte Sachverhalte im Kontext einer Erziehung zu thematisieren, die auf die Ermöglichung von Bildung gerichtet ist. Der besagte ,Konsens' ist benannt nach einem Tagungsort, an dem sich im Jahre 1976 die zu dieser Zeit führenden Politikdidaktiker im deutschsprachigen Raum eingefunden hatten, und markiert bis heute eine wichtige Zäsur in der Theorie des Politikunterrichts. Dabei ist festzuhalten, dass es sich nicht um einen offiziellen Beschluss der Tagung handelt, sondern um einen Tagungseindruck, der von einem der Teilnehmer, Hans-GeorgWehling, rückblickend formuliert worden ist. Aus der Sicht von Wehling waren es insbesondere ein Überwältigungsverbot 
- bisweilen auch Indoktrinationsverbot genannt - und ein Kontroversitätsgebot, worauf sich die Vertreter unterschiedlicher politikdidaktischer Positionen als Grundsätze des Politikunterrichts in modernen demokratischen Gesellschaften einigen konnten. Das Indoktrinationsverbot formuliert die Maßgabe, dass es "nicht erlaubt" sei, "den Schüler - mit welchen Mitteln auch immer - im Sinne erwünschter Meinungen zu überrumpeln und damit an der Gewinnung eines selbständigen Urteils zu hindern". Das Kontroversitätsgebot lautet: „Was in Wissenschaft und Politik kontrovers ist, muss auch im Unterricht kontrovers erscheinen". Der Schüler soll zur eigenen Urteilsbildung freigesetzt werden, und zwar dadurch, dass er gleichsam in den öffentlichen Streit hineingezogen wird. Den Schüler von diesem Streit fernzuhalten, indem „unterschiedliche Standpunkte unter den Tisch fallen, Optionen unterschlagen werden, Alternativen unerörtert bleiben", würde demgegenüber bedeuten, dass "der Weg zur Indoktrination beschritten" wird. ${ }^{32}$

In der Theorie nichtaffirmativer Erziehung sind das Indoktrinationsverbot und das Kontroversitätsgebot aufgegriffen und als allgemeine Prinzipien öffentlicher Erziehung ausgelegt worden. Dies mag zum einen damit zu tun haben, dass sich beide Prinzipien scheinbar nahtlos in den besagten Theorieansatz einfügen lassen. Zum anderen findet sich eine solche Verallgemeinerung bereits im Beutelsbacher Konsens angelegt, wird das Kontroversitätsgebot darin doch ausdrücklich nicht nur auf die Politik, sondern auch auf die Wissenschaft bezogen. Eine Erweiterung des Kontroversitätsgebots auf andere Bereiche erscheint von daher nur als konsequent, können Kontroversen in modernen demokratischen Gesellschaften doch nicht nur in wissenschaftlichen und politischen, sondern etwa auch in moralischen, religiösen oder wirtschaftlichen Fragen beobachtet werden. In diesem Sinne können wir etwa bei Benner lesen: Der Anspruch, dass Politikunterricht „plural und kontrovers diskutierte Fragen auch plural und kontrovers behandeln muss, lässt sich auch auf andere Fächer des schulischen Curriculums auslegen“. Hier wie dort liegen die Aufgaben öffentlicher Erziehung „nicht darin, die Schülerinnen und Schüler im Streit der Meinungen zu einem festen Urteil und einer entschiedenen Position zu führen, sondern ihnen Einsichten in die oft einander widerstreitenden Positionen zu vermitteln, Prozesse der Urteilsbildung anzustoßen, aber gerade nicht durch Belehrungen zum Abschluss zu bringen". ${ }^{33}$ Die Heranwachsenden sollen im Falle öffentlicher Kontroversen demnach in verschiedene Positionen eingeführt werden, und zwar so, dass sie die jeweiligen Positionen verstehen und im Streit der Positionen eigene Urteile entwickeln können, ohne sich hierbei auf eine definitive Position festlegen zu müssen. Der Prozess der Urteilsbildung soll vielmehr offen gehalten werden für Bildungsprozesse jenseits der Erziehung, in denen einmal gefundene Positionen erneut fraglich werden können und transformiert werden müssen.

\section{Ein blinder Fleck}

Erziehung in diesem Sinne kann mit Michael Hand als nichtdirektiv bezeichnet und von einer direktiven Erziehung unterschieden werden. "To teach a claim directively is to teach it with the intention of persuading students of its truth oder falsity; to teach a claim nondirectively is to teach it with the intention of not so persuading them"34. Direktive Erziehung ist daran erkennbar, dass ein Sachverhalt mit Überzeugungsabsicht thematisiert wird. Das bedeutet, ein Inhalt wird als geklärt behandelt. Der Erzieher versucht, den Heranwachsenden zu der einen richtigen 
Antwort auf eine bestimmte Frage zu führen. Der Zu-Erziehende soll sich die richtige Position aneignen. Nichtdirektive Erziehung bedeutet demgegenüber, einen Sachverhalt ohne Überzeugungsabsicht zu thematisieren. Das heißt, ein Inhalt wird als offen behandelt. Der Erzieher versucht, den Heranwachsenden in den Streit um die richtige Antwort auf eine bestimmte Frage einzuführen. Die verschiedenen Positionen, die in diesem Zusammenhang eingenommen werden, sollen dabei so neutral wie möglich behandelt werden. Der Zu-Erziehende soll die verschiedenen Positionen und ihre Begründungen verstehen sowie eine eigene Position im Lichte widerstreitender Alternativen entwickeln und diese gegen Einwände verteidigen können. ${ }^{35}$

Es ist wichtig zu betonen, dass die Differenz zwischen einer direktiven und einer nichtdirektiven Erziehung allein die Frage betrifft, ob ein Sachverhalt mit oder ohne Überzeugungsabsicht thematisiert wird. Das bedeutet, direktive und nichtdirektive Erziehung können in methodischer Hinsicht durchaus als variabel betrachtet werden. Ich werde an dieser Stelle jedoch nicht näher auf methodische Fragen der Thematisierung kontroverser bzw. nichtkontroverser Sachverhalte eingehen. Stattdessen möchte ich den Blick auf die Frage lenken „How are we to decide what to teach as controversial and what to teach as settled?"36. Diese Frage ist - wie man mit Hand diagnostizieren kann - „a difficult normative question"37, was sich nicht zuletzt daran zeigt, dass heute zwar eine Reihe von Antwortmöglichkeiten auf diese Frage existiert, keiner dieser Vorschläge bislang jedoch allgemeine Akzeptanz erfahren hat. In der Debatte um das ,beste' Kriterium ist bis dato kein Punkt erreicht worden, an dem ein Kriterium als unstrittig angesehen worden wäre. Darüber hinaus finden sich Stimmen, die gar die Sinnhaftigkeit der Debatte infrage stellen. ${ }^{38}$
Zugespitzt formuliert: Die Kontroverse um das Prinzip der Kontroversität besitzt selbst die Form einer in die Zukunft hinein offenen Debatte.

Die von Benner artikulierte Position, dass öffentliche Erziehung plural und kontrovers diskutierte Fragen auch plural und kontrovers behandeln müsse, erweist sich vor diesem Hintergrund nur auf den ersten Blick als plausibel und zeigt sich bei näherer Betrachtung durchaus als problembehaftet. Zwar wird in der Theorie nichtaffirmativer Erziehung Auskunft darüber gegeben, was es bedeutet, einen öffentlich kontrovers diskutierten Sachverhalt nichtdirektiv zu thematisieren. Es bleibt jedoch unklar, ob hier tatsächlich für die Position votiert wird, alle gesellschaftlich kontrovers diskutierten Sachverhalte auch im Kontext öffentlicher Erziehung als kontrovers zu thematisieren, oder ob hier nicht stillschweigend Kriterien in Anspruch genommen werden, die das Spektrum an entsprechenden Sachverhalten restringieren. So schreibt Benner in Bezug auf den Politikunterricht, dass der "Streit über politische Fragen" im Kontext einer nichtaffirmativen Erziehung offen gehalten werden müsse und „nicht mit Mitteln der Erziehung entschieden werden" dürfe. "Eine solche Erziehung", so Benner weiter, "hält sich nicht zu einer bestimmten Negation konkurrierender politischer Positionen befugt". ${ }^{39}$ An dieser Stelle kann jedoch gefragt werden, ob auch solche Sachverhalte als kontrovers thematisiert werden sollten, über die in der Öffentlichkeit nur deshalb eine Kontroverse besteht, weil hier Positionen eingenommen werden, die gegen die für moderne demokratische Gesellschaften maßgeblichen Werte der Freiheit, Gleichheit und Solidarität verstoßen - und damit gegen jene Werteorientierung, die überhaupt erst Anlass dazu gibt, den Edukanden als Subjekt zu adressieren und diesen (in einem anspruchsvollen Sinne) 
zur Selbsttätigkeit aufzufordern. Es wäre geradezu widersinnig, so könnte argumentiert werden, im Namen einer nichtaffirmativen Erziehung Positionen, die keine Wertschätzung von Pluralität zum Ausdruck bringen, als gleichwertige Alternativen zu Positionen zu thematisieren, für die eine Verpflichtung auf die Werteorientierung einer modernen demokratischen Gesellschaft charakteristisch ist. ${ }^{40}$ Das Kontroversitätsgebot würde in dieser Auslegung geradezu die Überwindung bestimmter Positionen verlangen.

Ich werde auf das hier nur skizzierte Argument noch näher eingehen. Worauf ich an dieser Stelle aufmerksam machen möchte, ist allein der Umstand, dass man bei einem Votum für ein Kontroversitätsgebot öffentlicher Erziehung nicht umhinkommt, zu der Frage Stellung zu nehmen, anhand welcher Kriterien unterschieden werden sollte, welche Sachverhalte direktiv und welche Sachverhalte nichtdirektiv thematisiert werden. Das Kontroversitätsgebot ist notwendigerweise mit der Frage nach entsprechenden Kriterien verknüpft. Selbst der ausdrückliche Verzicht auf ein solches Kriterium bedeutet, ein Kriterium in Anspruch zu nehmen, nämlich dass alle gesellschaftlich kontrovers diskutierten Sachverhalte auch im Kontext öffentlicher Erziehung als kontrovers thematisiert werden sollten. Das aber bedeutet zugleich, dass immer schon bestimmte Kriterien in Anspruch genommen werden, wenn überhaupt für ein Kontroversitätsgebot votiert wird. Diesen Umstand nicht zu berücksichtigen, ist der, blinde Fleck', der mit dem Kontroversitätsgebot im Kontext einer Theorie nichtaffirmativer Erziehung verknüpft ist. Dieser hat zur Folge, dass der Anspruch eines modernen Wissenschaftsverständnisses unterlaufen wird, die Voraussetzungen unserer Beschreibungen von Erziehung möglichst rational zu strukturieren. ${ }^{41}$ Stattdessen besteht die
Gefahr, dass Kriterien in Anspruch genommen werden, ohne diese einer kritischen Prüfung zu unterziehen. Ein solches Defizit kann nur behoben werden, indem Kriterien expliziert sowie auf ihre Vor- und Nachteile hin diskutiert werden.

In der Philosophy of Education wird zurecht darauf aufmerksam gemacht, dass es einen Unterschied macht, ob man z.B. ein soziales, ein politisches oder ein epistemisches Kriterium in Anspruch nimmt, um zwischen Sachverhalten zu unterscheiden, die direktiv bzw. nichtdirektiv thematisiert werden sollten. Es ist nun einmal etwas anderes, zu sagen, eine Frage sollte dann als kontrovers thematisiert werden, wenn diese in der Öffentlichkeit kontrovers diskutiert wird, wenn diese nicht in Bezug auf die öffentlichen Werte liberal-demokratischer Gesellschaften beantwortet werden kann, oder wenn diese Gegenstand vernünftiger Meinungsverschiedenheiten ist. ${ }^{42}$ Das Votum für ein soziales, politisches oder epistemisches Kriterium nimmt spezifische Voraussetzungen in Anspruch, zieht spezifische Begründungslasten nach sich und geht mit spezifischen Konsequenzen einher. In Bezug auf die hier behandelte Problemstellung folgt hieraus dreierlei: Erstens kann es keineswegs als selbstverständlich angesehen werden, welche Sachverhalte im Kontext öffentlicher Erziehung als kontrovers thematisiert werden sollten. Zweitens ist damit zu rechnen, dass man der Kriterienfrage prinzipiell nicht ausweichen kann, wenn man überhaupt für ein Kontroversitätsgebot votiert. Drittens kann nicht ohne Weiteres davon ausgegangen werden, dass bereits bekannte Kriterien sich nahtlos in die Theorie nichtaffirmativer Erziehung einfügen lassen. Vielmehr dürfte es erforderlich sein, das Problem der Kontroversität im Lichte der rekonstruierten Grundannahmen und -begriffe dieses Theorieansatzes ,eigenständig' zu bearbeiten, d.h. einen 
pädagogischen Vorschlag für ein entsprechendes Kriterium bzw. für entsprechende Kriterien zu entwickeln.

\section{Zwei Fragen}

Ich bin bereits auf das Prinzip der Nichthierachizität zu sprechen gekommen. Dieses verlangt freilich nicht nur, dass die Erziehung nicht für gesellschaftliche Zwecke instrumentalisiert werden darf, soll diese entsprechend ihrer Eigenlogik funktionieren können. Auch die Erziehung hat sich am Prinzip der Nichthierachizität auszurichten, d.h. auch sie darf gegenüber den jeweils anderen gesellschaftlichen Bereichen keinen Primat für sich in Anspruch nehmen.

Wenn es nun in der Wissenschaft, in der Politik, in der Religion, in der Moral oder zwischen einzelnen gesellschaftlichen Bereichen Streit gibt, dann - so scheint es jedenfalls - kann es nicht Aufgabe der Erziehung sein, den jeweiligen Streit zu entscheiden oder auch nur vorzuentscheiden, indem eine spezifische Perspektive als privilegiert behandelt wird. In diesem Fall würde die Erziehung nämlich - so könnte man meinen - eine übergeordnete Position für sich beanspruchen und damit gegen das Prinzip der Nichthierachizität verstoBen. Zugespitzt formuliert: Gibt es über einen Sachverhalt öffentlichen Streit, so scheint es die einzig legitime Aufgabe von Erziehung zu sein, Heranwachsende in den Streit einzuführen. Im Folgenden möchte ich zeigen, dass ein solcher Schluss voreilig wäre. Man übersieht dann nämlich, dass es gerade unter Rekurs auf die Eigenstruktur moderner Erziehung gute Gründe dafür gibt, nicht alle öffentlichen Kontroversen als gleichwertig zu behandeln und nur bestimmte Sachverhalte, die gesellschaftlich kontrovers diskutiert werden, auch im Kontext öffentlicher Erziehung als kontrovers zu thematisieren.
Mein Lösungsvorschlag zum Problem der Kontroversität sieht zunächst eine Differenz zwischen zwei Fragen vor, die in diesem Zusammenhang eine Rolle spielen, jedoch nicht immer mit hinreichender Klarheit auseinandergehalten werden. Die erste Frage lautet, welche Kontroversen im Kontext öffentlicher Erziehung überhaupt thematisiert werden sollten. Die zweite Frage heißt, welche dieser Kontroversen mit offenem Ausgang thematisiert werden sollten. Aus Platzgründen werde ich auf die erste Frage nur kurz eingehen. Dies nicht so sehr deshalb, weil diese Frage in der aktuellen Debatte über das Kontroversitätsgebot lediglich eine untergeordnete Rolle spielt (was eher ein Anlass dazu wäre, der Frage verstärkt Aufmerksamkeit zu widmen). Der Grund besteht vielmehr darin, dass es sich bei dieser Frage letztlich um einen Spezialfall einer allgemeineren Frage handelt - der Frage nämlich, welche Inhalte es überhaupt wert sind, dass sie im Kontext öffentlicher Erziehung thematisiert werden. Hinsichtlich dieser Frage steht - aus der Warte einer Theorie nichtaffirmativer Erziehung betrachtet - eine Reihe von gut begründeten Antworten bereit. So müssen Sachverhalte, die im Kontext öffentlicher Erziehung thematisiert werden, z.B. einen Bezug zur gegenwärtigen und zukünftigen Lebensführung des Einzelnen erkennen lassen. ${ }^{43}$ Kontroversen, die dieser Erwartung nicht entsprechen, sollten im Kontext öffentlicher Erziehung keinen Ort haben. In diesem Sinne dürfte es zweifelslos gerechtfertigt sein, die Kontroverse zwischen der Position, der Klimawandel sei menschengemacht, und der Position, der Klimawandel sei nicht menschengemacht, im Kontext öffentlicher Erziehung zu behandeln. Ob dies auch für Debatten gilt, in denen die Gestalt der Erde zur Disposition steht, mag hingegen mehr als bezweifelt werden. 
Zwar erlauben es die Kriterien der Gegenwarts- und Zukunftsbedeutung, die Fülle an potentiell thematisierbaren Kontroversen einzudämmen. Beide Kriterien geben hingegen keine Antwort auf die Frage, welche Sachverhalte im Kontext öffentlicher Erziehung direktiv bzw. nichtdirektiv behandelt werden sollten. Hierzu bedarf es zusätzlicher Kriterien wobei auch in diesem Falle gilt, dass die Rechtfertigung relational zu den Grundannahmen und -begriffen einer Theorie nichtaffirmativer Erziehung erfolgen muss.

\section{Wider dem sozialen Kriterium}

Nichtaffirmative Erziehung ist durch den Anspruch gekennzeichnet, Bildung zu ermöglichen. Dies bedeutet zum einen, den Einzelnen mit einer widerständigen Welt zu konfrontieren. Zum anderen bedeutet dies, dem Einzelnen die Möglichkeit zu eröffnen, sich zu dieser Welt zu verhalten. Ein solcher Begriff von Erziehung ist - so meine These - prinzipiell inkompatibel mit der Auffassung, dass alle öffentlich kontrovers diskutierten Sachverhalten im Kontext von Erziehung als kontrovers thematisiert werden sollten. Zugespitzt formuliert: Im Kontext einer Theorie nichtaffirmativer Erziehung kann nicht sinnvoll für ein soziales Kriterium argumentiert werden.

Für die Zurückweisung eines sozialen Kriteriums lassen sich mindestens zwei Argumente anführen. Ein erstes Argument ergibt sich dann, wenn man den mit nichtaffirmativer Erziehung verbundenen normativen Anspruch berücksichtigt, jeden Einzelnen als Selbstzweck zu achten und diesen deshalb im Kontext von Erziehung als Subjekt zu adressieren. Diese normative Orientierung würde gleichsam ad adsurdum geführt werden, wenn für ein soziales Kriterium votiert wird, müssten in diesem Fall doch auch solche Kontroversen nichtdirektiv thematisiert werden, die nur deshalb bestehen, weil in der Öffentlichkeit Positionen vertreten werden, die der besagten normativen Orientierung zuwiderlaufen.

Ein zweites Argument, das gegen ein soziales Kriterium spricht, ergibt sich daraus, dass Bildung ein Sich-Verhalten zu einer widerständigen Welt bedeutet. Nichtaffirmative Erziehung schließt vor diesem Hintergrund immer auch mit ein, auf der Seite der Heranwachsenden Differenzerfahrungen zu provozieren - etwa dadurch, dass dem Einzelnen nicht der Gefallen getan wird, jede Position als gleichermaßen gültig zu akzeptieren. Die für Bildungsprozesse charakteristische Widerständigkeit tritt in diesem Fall dadurch in Erscheinung, dass der Erzieher eine bestimmte Position als die richtige Position in Anschlag bringt - was nicht notwendigerweise bedeutet, dass dem Einzelnen keine Möglichkeit des Sich-Verhaltens eingeräumt wird oder eingeräumt werden könnte. Der Erzieher eröffnet einen entsprechenden Freiraum des Sich-Verhaltens z.B. dadurch, dass er Gründe anführt, warum eine bestimmte Position vorgezogen werden sollte, und die Heranwachsenden zu deren Prüfung auffordert. Direktiv zu erziehen, ist in diesem Sinne durchaus kompatibel damit, den Einzelnen als Subjekt zu adressieren. Den Einzelnen zur Prüfung von Geltungsansprüchen aufzufordern, impliziert nämlich immer auch die Möglichkeit, dass dieser einen Geltungsanspruch zurückweist. Umgekehrt ermöglicht es die Aufforderung zur Prüfung von Geltungsansprüchen, dass der Einzelne im Durcharbeiten von Differenzerfahrungen Positionen aus Einsicht zustimmt bzw. ein bestimmtes Wissen selber denkend ,hervorbringt". Direktive Erziehung lässt sich vor diesem Hintergrund als eine Form von Erziehung verstehen, in der Heranwachsende mit einer Widerständigkeit konfrontiert werden, die gerade darin besteht, dass gute Gründe 
dafür sprechen, einen Sachverhalt nicht als kontrovers anzusehen - mögen manche Personen auch anderer Überzeugung sein. Anhand eines sozialen Kriteriums zu unterscheiden, welche Sachverhalte direktiv bzw. nichtdirektiv thematisiert werden sollten, würde hingegen die Gefahr heraufbeschwören, dass Heranwachsende Positionen als umstritten betrachten oder gar als, richtig' akzeptieren, die es - auf dem heutigen Stand der Erkenntnis - nicht sind. Bildung aber heißt, sich zur Welt in ein Verhältnis zu setzen. Hierzu bedarf es der Einsicht darin, wie es sich mit den Sachen verhält - was eine skeptische Restriktion von Wissen auf bestimmte Voraussetzungen nicht aus-, sondern einschließt. ${ }^{44}$

Beide Argumente sprechen gegen ein soziales Kriterium und damit zugleich für eine spezifische Umgrenzung der im Kontext öffentlicher Erziehung als kontrovers zu behandelnden Sachverhalte. Mein Vorschlag sieht eine Kombination zweier Kriterien vor, die jeweils von der Eigenlogik moderner Erziehung her gerechtfertigt werden können. Demnach sollten nur solche Sachverhalte im Kontext öffentlicher Erziehung als kontrovers thematisiert werden, im Falle derer erstens Positionen miteinander konfligieren, die eine spezifische Werteorientierung akzeptieren, sowie zweitens eine übergeordnete Urteilsregel fehlt, die es erlauben würde, eine Position als die allein richtige auszuweisen. Werfen wir im Folgenden einen genaueren Blick auf diese Kriterienkombination.

\section{Ein demokratischer Rahmen}

Eine nichtaffirmative Erziehung ist auf die Ermöglichung von Bildung gerichtet. Es wäre vor diesem Hintergrund widersinnig, auf der einen Seite für eine Freisetzung der Heranwachsenden zu eigener Urteilsbildung zu votieren, und gleichzeitig
Positionen als gleichberechtigt oder auch nur als diskussionswürdig zu behandeln, deren normative Orientierung mit der einer Erziehung als Ermöglichung von Bildung inkompatibel ist. Zugespitzt formuliert: Wenn man eingesehen hat, dass eine nichtaffirmative Erziehung auf spezifischen normativen Voraussetzungen beruht, dann versteht man auch, dass es diese Prämissen letztlich unterminieren würde, wollte man tatsächlich alles, was in einer Gesellschaft kontrovers diskutiert wird, auch im Kontext öffentlicher Erziehung als kontrovers thematisieren. Man müsste dann nämlich die Frage beantworten, warum man überhaupt für eine Erziehung als Ermöglichung von Bildung votiert, wenn man doch gleichzeitig dazu bereit ist, den damit verbundenen normativen Anspruch, jeden Einzelnen als Selbstzweck zu betrachten und zu behandeln, zu relativieren, indem Positionen als gleichberechtigt behandelt werden, die mit einer solchen Orientierung inkompatibel sind. Das Kontroversitätsgebot verlangt so gesehen nicht nur, dass bestimmte öffentliche Kontroversen auch im Kontext von Erziehung als kontrovers thematisiert werden. Das Kontroversitätsgebot verlangt auch danach, dass in diesem Zusammenhang bestimmte Positionen entweder außen vor bleiben oder aber der Kritik ausgesetzt werden.

Diese Position lässt sich weiter untermauern, wenn man nicht nur die normativen Prämissen einer Erziehung als Ermöglichung von Bildung berücksichtigt, sondern darüber hinaus auch den Umstand, dass Bildungsprozesse selbst in spezifischer Hinsicht normativ umgrenzt sind. Bildung - so habe ich deutlich zu machen versucht - bezeichnet den Prozess, in dem der Einzelne die Fähigkeit entwickelt, sein Leben selbstbestimmt zu führen. Selbstbestimmungsfähigkeit darf in diesem Zusammenhang nicht - und das ist hier entscheidend - individualistisch missverstanden werden. Diese ist vielmehr an 
den Anspruch geknüpft, dass der Einzelne die Fähigkeit und Bereitschaft entwickelt, auch allen anderen Menschen die Freiheit einzuräumen bzw. allererst zu ermöglichen, ihr Leben selbstbestimmt zu führen. Geht man von der Annahme aus, dass Bildung in der Entwicklung von Selbstbestimmungsfähigkeit besteht, und geht man ferner davon aus, dass die selbstbestimmte Lebensführung einen Freiraum benötigt, der im Miteinanderumgehen von Menschen nicht notwendigerweise gegeben ist, sondern allererst hergestellt und stabilisiert werden muss, dann folgt hieraus, dass es verkürzt wäre, Bildung allein als Entwicklung von Selbstbestimmungsfähigkeit zu begreifen. Bildung wird konsequenterweise auch als die Entwicklung der Fähigkeit und Bereitschaft begriffen werden müssen, anderen Menschen ebenfalls die Freiheit zur selbstbestimmten Lebensführung zuzugestehen bzw. allererst zu ermöglichen - dies jedenfalls dann, wenn die Entwicklung von Selbstbestimmungsfähigkeit als ein Anspruch aller Menschen aufgefasst wird.

Fasst man die Freiheit, auf deren Ermöglichung Erziehung bezogen ist, nicht vorschnell als "neo-liberal-freedom", sondern eben als "democratic freedom" auf ${ }^{45}$, so kann also auch vor diesem Hintergrund für eine spezifische Auslegung des Kontroversitätsgebots öffentlicher Erziehung argumentiert werden. Es wäre nämlich nicht minder widersinnig, wenn Bildung eine Entwicklung der Haltung impliziert, andere Menschen als Selbstzweck zu betrachten und zu behandeln, im Kontext öffentlicher Erziehung aber Positionen, die diesem Anspruch entsprechen, gleichberechtigt neben Positionen thematisiert werden, die den besagten Anspruch unterlaufen, d.h. Menschen nicht die gleiche Freiheit einräumen, eigene Positionen zu finden, zu leben und weiterzuentwickeln.

\section{Komplexität}

Es stellt sich die Frage, ob es im Kontext einer Theorie nichtaffirmativer Erziehung Gründe dafür gibt, das Spektrum der nichtdirektiv zu thematisierenden Sachverhalte noch weiter einzugrenzen. Werfen wir hierzu zunächst einen Blick auf Beispiele für Kontroversen, von denen im Rahmen dieses Theorieansatzes behauptet wird, sie sollten mit offenem Ausgang thematisiert werden. Hierzu zählen erstens Kontroversen zwischen politischen Positionen, in denen Interessen der Gesellschaftsbewahrung auf Interessen der Gesellschaftsveränderung stoßen ${ }^{46}$, zweitens Konflikte zwischen wissenschaftlichen Paradigmen, die mit unterschiedlichen methodischen Leitfragen operieren und - damit verbunden - verschiedene Wissensformen hervorbringen, sowie drittens Konflikte zwischen verschiedenen gesellschaftlichen Bereichen wie Politik, Wissenschaft, Religion, Moral, Kunst, Wirtschaft und Erziehung, die jeweils nach einer eigenen Logik operieren. ${ }^{47}$

Gibt es eine Gemeinsamkeit zwischen diesen verschiedenen Arten von Konflikten? Mir scheint eine solche darin zu bestehen, dass jeweils eine übergeordnete Regel fehlt, die den Vorzug einer der miteinander konkurrierenden Positionen - und damit zugleich die Zurückstellung aller restlichen Optionen - allgemeinverbindlich rechtfertigen könnte. Entsprechend besteht jeweils die Möglichkeit, eine Position mit Alternativen zu konfrontieren, für die ebenfalls gute Gründe sprechen, ohne dass ein bester Grund zur Verfügung steht, der eine Wahl gleichsam ,hieb- und stichfest' rechtfertigen könnte.

Um die Eigenart solcher Kontroversen genauer zu fassen, erweist sich aus meiner Sicht die Unterscheidung zwischen Einfachheit, Kompliziertheit und Komplexität als hilfreich, wie sie in der Komplexitätstheorie eingeführt worden ist. ${ }^{48}$ Einfache 
und komplizierte Problemstellungen unterscheiden sich von komplexen Problemstellungen dadurch, das Regeln bekannt sind, um ein gegebenes Problem erwartbar erfolgreich zu lösen. Im Falle von einfachen Problemstellungen können die zur Problemlösung erforderlichen Regeln störungsfrei eingesetzt werden. Im Falle von komplizierten Problemstellungen ist der störungsfreie Einsatz von Regeln hingegen nicht möglich, was z.B. daran liegen kann, dass Personen die entsprechenden Regeln nicht kennen. In diesem Sinne mag ein bestimmtes Problem für Experten als einfach, für Laien hingegen als kompliziert erscheinen. Im Unterschied hierzu zeichnen sich komplexe Problemstellungen dadurch aus, dass keine Regeln bekannt sind, die es erlauben würden, ein gegebenes Problem erwartbar erfolgreich zu lösen. Dies gilt - und das ist hier entscheidend - auch auf der Seite der Experten.

Operiert man mit der Differenz zwischen Einfachheit, Kompliziertheit und Komplexität, so ist es möglich, zwischen verschiedenen Arten von Kontroversen zu unterscheiden. So können auf der einen Seite Kontroversen ausgemacht werden, zu deren Auflösung auf eine übergeordnete Regel rekurriert werden kann, um festzustellen, welche Antwort auf eine bestimmte Frage die richtige ist. Auf der anderen Seite gibt es Kontroversen, in denen verschiedene Positionen aufeinandertreffen, ohne dass die Möglichkeit besteht, auf eine entsprechende Regel Bezug zu nehmen. Eine solche Kontroverse erweist sich in einem spezifischen Sinne als irreduzibel. Zugespitzt formuliert: Im Falle von komplexen Problemstellungen gibt es keine allgemeingültige Antwort auf die Frage, worin die allein richtige Position besteht, die im Verhältnis zu einem Sachverhalt eingenommen werden sollte. Umgekehrt bedeutet dies, dass wir es mit verschiedenen Positionen zu tun haben, für die jeweils gute Gründe angeführt werden können. ${ }^{49}$
Im Lichte der Differenz zwischen einfachen, komplizierten und komplexen Problemstellungen ist es möglich, das Kriterium genauer zu fassen, das in der Theorie nichtaffirmativer Erziehung implizit eine Rolle zu spielen scheint, um zwischen Sachverhalten, die direktiv, und solchen Sachverhalten, die nichtdirektiv thematisiert werden sollten, zu unterscheiden. Diesem Kriterium zufolge sollten Fragen als nichtdirektiv thematisiert werden, bei denen es sich um komplexe Problemstellungen handelt. Als direktiv wären demgegenüber solche Fragen zu thematisieren, die lediglich kompliziert sind. Etwaige Kontroversen, die auch im Falle von komplizierten Fragen beobachtbar sind, können in der Erziehung freilich aufgegriffen werden - jedoch nicht so, als wären die jeweils konfligierenden Positionen als gleichberechtigt anzusehen. Im Falle von komplizierten Fragen ist nichtaffirmative Erziehung nicht darauf gerichtet, Heranwachsende zu eigener Urteilsbildung aufzufordern, sondern vielmehr von der Intention getragen, dem Einzelnen Einsicht in die richtige Antwort auf eine Frage zu ermöglichen. ${ }^{50}$

Allein der Umstand, dass in der Theorie nichtaffirmativer Erziehung offenbar komplexe Problemstellungen im Fokus stehen, wenn für ein Kontroversitätsgebot öffentlicher Erziehung votiert wird, besagt freilich noch nicht, ob und, falls ja, wie ein solches Kriterium in diesem Kontext begründet werden kann. Dieser Frage möchte ich mich nun abschließend zuwenden, wobei ich zu zeigen versuche, dass es gleichermaßen problematisch wäre, offene Sachverhalte als geklärt und geklärte Sachverhalte als offen zu thematisieren.

Eine Erziehung, die Sachverhalte als geklärt behandelt, obgleich es alternative Positionen gibt, für die ebenfalls gute Gründe sprechen, ist mit dem Anspruch nichtaffirmativer Erziehung, Bildung zu ermöglichen, inkompatibel. In diesem Fall kann Erziehung nämlich nicht konsequent 
dialogisch, d.h. als Aufforderung zur Prüfung von Geltungsansprüchen praktiziert werden. Da im Falle von komplexen Problemstellungen keine Position mit einem Argument aufwarten kann, das absolute Durchschlagskraft besitzt, wird eine solche Erziehung zu Methoden und Mitteln greifen müssen, die das Sich-Verhalten des Einzelnen unterbinden, um diesen dazu zu veranlassen, eine bestimmte Position für gerechtfertigt zu halten (Alternativen werden verschwiegen, Positionen werden ,verzerrt' dargestellt, eine Person soll mittels Sympathie zur Übernahme einer Position bewogen werden etc.). Bildung bedeutet aber gerade nicht, sich auf Positionen in einer Art und Weise zu verpflichten, in der das Sich-Verhalten des Einzelnen suspendiert ist. Positiv formuliert: Um Bildung zu ermöglichen, ist es geboten, dass Kontroversen, in denen Positionen eingenommen werden, für die jeweils gute Gründe sprechen, nicht vorentschieden werden, sondern der Erzieher dem Edukanden dabei hilft, „einen eigenen Weg zu finden und zu gehen"51.

Eine Erziehung, die Sachverhalte als offen behandelt, obgleich es eine Position gibt, die allen anderen Positionen überlegen ist, unterläuft ebenfalls den Anspruch, den Einzelnen für ein Leben in Selbstbestimmung freizusetzen. Zum einen besteht die Gefahr, dass der Einzelne sich falsche Meinungen aneignet, zum anderen besteht die Gefahr, dass der Einzelne glaubt, es gäbe in Bezug auf einen bestimmte Frage keine vorziehenswürdige Antwort. In beiden Fällen wird die Bedeutung eines bereits erreichten Erkenntnisstandes - einer "geschichtlich erreichten Gedanklichkeit"52 - unterminiert, die diesem in Kontext einer Erziehung als Ermöglichung von Bildung zukommt. Der Einzelne soll dazu freigesetzt werden, sich im Verhältnis zur Welt selbst zu bestimmen. Wissen nennen wir solche Beschreibungen, die bis dato der kritischen Prüfung standgehalten haben, so dass wir - aktuell jedenfalls keinen Anlass dazu haben, die Geltung der jeweiligen Beschreibungen in Frage zu stellen. Kurzum: Eine Erziehung, die nichtdirektiv ausgerichtet ist, obgleich Sachverhalte thematisiert werden, die als geklärt betrachtet werden können, geht zumindest mit der Gefahr einher, Heranwachsende von der Welt zu ,entfernen' und dazu beizutragen, dass diese ihr Leben eingesponnen in Täuschungen führen lernen. Dies wäre nicht nur in moralischer Hinsicht problematisch, kann dies doch einen beträchtlichen Schaden auf der Seite des Heranwachsenden zur Folge haben ("Ich wusste nicht, dass Rauchen das Risiko erhöht, an Lungenkrebs zu erkranken."). Darüber hinaus würde damit ein Anspruch unterlaufen werden, der traditionell mit dem Bildungsbegriff markiert wird, nämlich dass Heranwachsende die Fähigkeit entwickeln, im Lichte sachlicher Einsichten Stellung zu nehmen. Bildung bedeutet insofern gerade nicht, in Täuschungen befangen zu sein. Positiv formuliert: Es ist aus pädagogischer Warte geboten, dass der Erzieher die Intention verfolgt, Heranwachsende davon zu überzeugen, dass eine bestimmte Position die richtige Position ist, wenn diese Position die einzige ist, für die heute gute Gründe angeführt werden können.

\section{Noch einmal: Das Prinzip der Nichthierachizität}

Bleibt abschließend zu fragen: Konfligiert der hier entwickelte Vorschlag nicht mit dem Prinzip der Nichthierachizität? Besagt dieses Prinzip nicht, dass öffentlicher Streit nicht mit den Mitteln der Erziehung entschieden werden dürfe? Wird der Erziehung hier nicht ein Primat zugesprochen, der dieser nicht zukommt?

Bezogen auf die von mir vorgeschlagenen Kriterien könnte ein erster Einwand 
in der Tat lauten, der Erziehung stehe es nicht zu, öffentliche Kontroversen relational zu bestimmten normativen Prämissen aufzulösen. Die Wahl der eigenen Lebensform dürfe im Kontext öffentlicher Erziehung nicht vorentschieden werden. Einem solchen Einwand kann entgegengehalten werden, dass dieser selbst einen normativen Anspruch zum Ausdruck bringt, nämlich im Kontext öffentlicher Erziehung die Möglichkeit offenzuhalten, dass der Einzelne seine Lebensform selbst wählt. Nichts anderes aber ist mit dem Bildungsanspruch nichtaffirmativer Erziehung gemeint. Es wäre nun aber geradezu absurd, im Namen der Selbstbestimmung für einen gleichberechtigten Umgang mit Positionen zu votieren, die dieser Idee ablehnend gegenüberstehen.

Ein zweiter Einwand könnte lauten, dass es der Erziehung nicht zustehe, Kontroversen relational zu dem Anspruch aufzulösen, dass allein ,vernünftige Meinungsverschiedenheiten' als kontrovers thematisiert werden sollten. Diesem Einwand wäre entgegenzuhalten, dass die Erziehung nur dann einen Primat für sich beanspruchen würde, wenn versucht wird, Kontroversen aufzulösen, in denen keine Position einen Primat für sich beanspruchen kann. In all den Fällen hingegen, in denen klar ist, welche Position vorzuziehen bzw. zurückzustellen ist - eben weil eine übergeordnete Urteilsregel zur Verfügung steht -, verstößt die Erziehung nicht gegen das Prinzip der Nichthierarchizität. Die hierarchische Ordnung der verschiedenen Positionen wird hier nämlich nicht durch die Erziehung hervorgebracht. Diese Ordnung ist vielmehr bereits, gegeben', und die Erziehung erfüllt nicht mehr als die Funktion, die Heranwachsenden mit diesem Umstand zu konfrontieren.

Das Prinzip der Nichthierarchizität erweist sich so gesehen als durchaus kompatibel mit der hier vorgeschlagenen Kriterienkombination. Es verlangt nicht danach, alle Sachverhalte, die gesellschaftlich kontrovers diskutiert werden, im Kontext öffentlicher Erziehung so zu thematisieren, als stünde die Antwort auf eine bestimmte Frage nicht schon fest. Im Kontext öffentlicher Erziehung sollten diejenigen - aber auch nur diejenigen - Sachverhalte mit offenem Ausgang thematisiert werden, im Falle derer Positionen konfligieren, die den Anspruch von Menschen auf wechselseitige Achtung ihrer Freiheit akzeptieren, und darüber hinaus keine übergeordnete Regel bekannt ist, um die jeweiligen Positionen in die allein ,richtige‘ Ordnung zu überführen.

\section{Anmerkungen}

1 Vgl. Benner, D. (1982): Bruchstücke zu einer nicht-affirmativen Theorie pädagogischen Handelns. In: Zeitschrift für Pädagogik 28(6), S. 952-967; Benner, D. (1988/1995): Bildsamkeit und Bestimmung. Zu Fragestellung und Ansatz nicht-affirmativer Bildungstheorie. In: D. Benner: Studien zur Theorie der Erziehung und Bildung. Weinheim und München: Juventa, S. 141-159; Benner, D. (2015): Allgemeine Pädagogik. Eine systematischproblemgeschichtliche Einführung in die Grundstruktur pädagogischen Denkens und Handelns. 8. Auflage. Weinheim und Basel: Beltz Juventa. Zur internationalen Rezeption vgl. Uljens, M. (2002): The Idea of a Universal Theory of Education - an Impossible but Necessary Project? In: Journal of Philosophy of Education 36(3), S. 353-375; Uljens, M./ Ylimaki, R. M. (2017): Non-affirmative Theory of Education as a Foundation for Curriculum Studies, Didaktik and Educational Leadership. In: M. Uljens and R. M. Ylimaki (eds.): Bridging Educational Leadership, Curriculum Theory and Didaktik. Non-affirmative Theory of Education. Dordrecht: Springer, S. 3-145.

2 Uljens, M. (2018a): A Contribution to Re-theorizing Curriculum Research. In: Transnational Curriculum Inquiry 15(2), S. 4-25, hier: S. 22.

3 Vgl. Brüggen, F. (1997): Bildsamkeit und Mündigkeit des Subjekts. Bildungsgeschichtliche und bildungstheoretische Überlegungen 
zu einer (nicht nur) pädagogischen Idee. In: Franz Fischer Jahrbuch für Philosophie und Pädagogik, S. 111-125, hier: S. 115ff.

4 Vgl. Luhmann, N. (1997): Die Gesellschaft der Gesellschaft. Frankfurt am Main: Suhrkamp; Nassehi, A. (2017): Die letzte Stunde der Wahrheit. Kritik der komplexitätsvergessenen Vernunft. Hamburg: Murmann.

5 Zur besseren Lesbarkeit verwende ich in diesem Beitrag das generische Maskulinum.

6 Vgl. Herbart, J. F. (1806/1964): Allgemeine Pädagogik aus dem Zweck der Erziehung abgeleitet. In: Joh. Fr. Herbart's Sämtliche Werke in chronologischer Reihenfolge. Hrsg. v. K. Kehrbach u. O. Flügel. Band 2. Aalen: Scientia, S. 1-139, hier: S. 110ff.; Schleiermacher, F. (1826/2000): Grundzüge der Erziehungskunst. In: F. Schleiermacher Texte zur Pädagogik Hrsg. v. M Winkler u. J. Brachmann. Band 2. Frankfurt am Main: Suhrkamp, hier: S. 75.

7 Vgl. Hand, M. (2008): What Should We Teach As Controversial? A Defense of the Epistemic Criterion. In: Educational Theory 58(2), S. 213-228; Cooling, T. (2012): What is a Controversial Issue? Implications for the Treatment of Religious Beliefs in Education. In: Journal of Beliefs and Values 33(2), S. 169-181; Gregory, M. R. (2014): The Procedurally Directive Approach to Teaching Controversial Issues. In: Educational Theory 64(6), S. 627-649; Warnick, B. R./ Spencer Smith, D. (2014): The Controversy over Controversies: A Plea for Flexibility and for "Soft-directive" Teaching. In: Educational Theory 64(3), S. 227-244; Hess, D. E./McAvoy, P. (2014): The Political Classroom: Evidence and Ethics in Democratic Education. New York: Routledge; Tillson, J. (2017): When to Teach for Belief: A Tempered Defense of the Epistemic Criterion. In: Educational Theory 67(2), S. 173-191; Zimmerman, J./Robertson, E. (2017): The Case for Contention. Teaching Controversial Issues in American Schools. Chicago and London: University of Chicago Press; Yacek, D. (2018): Thinking Controversially: The Psychological Condition for Teaching Controversial Issues. In: Journal of Philosophy of Education 52(1), S. 71-86; Saetra, E. (2019): Teaching Controversial Issues: A Pragmatic View of the Criterion Debate. In: Journal of Philosophy of Edcation 53(2), S. 323-339. - Zur bislang nur sporadischen Rezeption der internationalen Debatte im deutschsprachigen Raum vgl. Drerup, J. (erscheint 2021): Demokratieerziehung und die Kontroverse über Kontroversitätsgebote. In: Zeitschrift für Pädagogik 67(4); Giesinger, J. (erscheint 2021): Kontroversität im Ethikunterricht: Das Kriterium der öffentlichen Rechtfertigbarkeit. In: M. Kim/K. Neeg/J. F. Friedrich/T. Gutmann (Hrsg.): Werte im Ethikunterricht. An den Grenzen der Wertneutralität. Opladen: Barbara Budrich.

8 Vgl. Hand 2008.

9 Vgl. Biesta, G. J. J. (2013): On the Idea of Educational Theory. In: B. Irby/G. Brown/R. Lara-Alecio/S. Jackson (Hrsg.): The Handbook of Educational Theories. Charlotte, NC: Information Age Publishing, S. 5-15, hier: S. 13.

10 Vgl. Benner 2015, S. 30ff.

11 Vgl. Sünkel, W. (2013): Erziehungsbegriff und Erziehungsverhältnis. Allgemeine Theorie der Erziehung. Weinheim und Basel: Beltz Juventa, S. 29ff u. 88ff.

12 Vgl. Winkler, M. (2006): Kritik der Pädagogik. Der Sinn der Erziehung. Stuttgart: Kohlhammer, S. $71 \mathrm{ff}$.

13 Benner, D. (1979): Lässt sich das Technologieproblem durch eine Technologieersatztechnologie lösen? In: Zeitschrift für Pädagogik 25(3), S. 367-375, hier: S. 369f. (Herv. d. Verf.).

14 Vorläufer einer Beschreibung von Erziehung als nichtaffirmativ können bis in die Antike zurückverfolgt werden.

15 Vgl. Benner 1988/1990, S. $98 f$.

16 Uljens, M. (2018b): Understanding Educational Leadership and Curriculum Reform: Beyond Global Economism and Neo-Conservative Nationalism. In: Nordic Journal of Comparative and International Education 2(2/3), S. 196-213, hier: S. 205.

17 Benner 2015, S. 171.

18 Heitger, M. (1984): Der Begriff der Bildung unter den institutionellen Bedingungen von Schule. In: M. Heitger (Hrsg.): Umgang mit der Schulkritik. Münster: Aschendorff, S. 32-47, hier: S. 42ff.

19 Ebd.

20 Brüggen, F. (1999): Bildung als Orientierungsform. Bemerkungen zur Stellung der Bildung zwischen Ethik und Wissenschaft. In: A. Wenger-Hadwig (Hrsg.): Verführung in orientierungsloser Zeit. Innsbruck und Wien: Tyrolia, S. 43-66, hier: S. 60.

21 In der Sprache der Komplexitätstheorie könnte man an dieser Stelle auch von 
vorübergehend stabilen Gleichgewichtszuständen sprechen.

22 Vgl. Sünkel 2013, S. 93ff. u. 100f.

23 Vgl. Biesta, G. J. J. (2020) Can the prevailing description of educational reality be considered complete? On the Parks-Eichmann paradox, spooky action at a distance, and a missing dimension in the theory of education. In: Policy Futures in Education. 18(4), S. 1011-1025.

24 Anhalt, E. (1999): Bildsamkeit und Selbstorganisation. Johann Friedrich Herbarts Konzept der Bildsamkeit als Grundlage einer pädagogischen Theorie der Selbstorganisation organismischer Aktivität. Weinheim: Deutscher Studienverlag.

25 Vgl. Mikhail, Th. (2016): Pädagogisch handeln. Theorie für die Praxis. Paderborn: Schöningh, S. $129 f f$.

26 Brüggen, F. (2009): Bildsamkeit und Mündigkeit als pädagogische Begriffe. In: Pädagogikunterricht 29(2/3), S. 4-11, hier: S. 4.

27 Vgl. Benner 2015, S. 108ff.

28 Schweitzer, F. (2019): Mündigkeit durch religiöse Bildung? Pädagogische Rundschau 73(5), S. 471-486, hier: S. 476ff.

29 Vgl. Benner 2015, S. 118ff.

30 Vgl. Uljens/Ylimaki 2017, S. $9 f$.

31 Für eine Verteidigung dieser Idee vgl. Rucker, Th. (2019): Erziehender Unterricht, Bildung und das Problem der Rechtfertigung. In: Zeitschrift für Erziehungswissenschaft 22(3), S. 647-663, hier: S. 657ff.

32 Für alle Zitate: Wehling, H.-G. (1977): Konsens à la Beutelsbach? In: S. Schiele/H. Schneider (Hrsg.): Das Konsensproblem in der politischen Bildung. Stuttgart: Klett, S. 173-184, hier: S. 179.

33 Benner, D. (2018): Religion im Kontext öffentlicher Bildung und Erziehung. In: S. Müller/W. Sander (Hrsg.): Bildung in der postsäkularen Gesellschaft. Weinheim and Basel: Beltz Juventa, S. 144-162, hier: S. 155f.

34 Hand, M. (2014): Afterwords: Response to Warnick and Smith. In: Educational Theory 64(4), S. 425-426, hier: S. 425.

35 Vgl. Hand 2008, S. 213.

36 Ebd.

37 Ebd.

38 Vgl. Saetra 2019

39 Benner 2015, S. 324

40 Vgl. Sander, W. (2009): Bildung und Perspektivität. Kontroversität und Indoktrinationsverbot als Grundsätze von Bildung und
Wissenschaft. Erwägen Wissen Ethik 20(2), S. 239-248, hier: S. 247.

41 Vgl. Rucker, Th. (2017): Allgemeine Didaktik als Reflexionsinstanz. Versuch einer wissenschaftstheoretischen Grundlegung. In: Zeitschrift für Pädagogik 63(5), S. 618-635, hier: S. $626 \mathrm{ff}$.

42 Für einen Überblick vgl. Yacek 2018, S. 73ff.

43 Zur Rechtfertigung beider Kriterien vgl. Rucker, Th. (2020): Teaching and the Claim of Bildung: The View from General Didactics. Studies in Philosophy and Education 39(1), S. 51-69, hier: S. 62ff. Eine ähnliche Überlegung findet sich bei Robert Dearden, der festhält, dass bestimmte Kontroversen auf "matters of very great importance" bezogen seien, und diese Kontroversen es deshalb wert wären, thematisiert zu werden. Dearden, R. F. (1981): Controversial Issues and the Curriculum In: Journal of Curriculum Studies 13(1), S. 37-44, hier: S. 41). - Das Argument, das Dearden anführt, um zu rechtfertigen, warum kontroverse Sachverhalte überhaupt im Kontext öffentlicher Erziehung thematisiert werden sollten, kann wie folgt rekonstruiert werden: Wenn Erziehung darauf gerichtet ist, Heranwachsende in eine Gesellschaft einzuführen, diese aber durch öffentliche Kontroversen gekennzeichnet ist, so muss Erziehung Heranwachsende grundsätzlich auch in Kontroversen einführen. Dies gilt ebenso für die Wissenschaften sowie für schulische Fächer, die an bestimmten Wissenschaftsdisziplinen Orientierung finden. Zugespitzt formuliert: "To teach a subject in a way that makes no reference to the controversial parts of it is to misrepresent it", denn "at the cutting-edge of any subject, much, if not everything, will be controversial" (ebd.).

44 Vgl. Rucker 2020, S. 62.

45 Biesta, G. J. J. (2017): The Rediscovery of Teaching. New York and London: Routledge, S. 79 u. $7 f f$.

46 und die - das müsste nun freilich hinzugefügt werden - eine Verpflichtung auf die demokratischen Werte der Freiheit, Gleichheit und Solidarität erkennen lassen.

47 Vgl. Benner D./Stepkowski, D. (2012): Warum Erziehung in Demokratien nicht politisch fundiert werden kann. In: E. Anhalt/D. Stepkowski (Hrsg.): Erziehung und Bildung in politischen Systemen. Jena, Garamond, S. 49-71, hier: S. 68ff.; Benner, D. (2020): Umriss der allgemeinen 
Wissenschaftsdidaktik. Grundlagen und Orientierungen für Lehrerbildung, Unterricht und Forschung. Weinheim und Basel: Beltz Juventa, S. 76ff.; Benner 2015, S. 187.

48 Vgl. Rucker, Th./Anhalt, E. (2017): Perspektivität und Dynamik. Studien zur erziehungswissenschaftlichen Komplexitätsforschung. Weilerswist: Velbrück, S. 23ff.

49 An dieser Stelle zeigt sich unverkennbar eine Nähe der Überlegungen zu dem, was im Kontext der Philosophy of Education als epistemisches Kriterium diskutiert wird: Dieses Kriterium besagt, dass nur solche Sachverhalte im Kontext öffentlicher Erziehung als kontrovers thematisiert werden sollten, die
Gegenstand vernünftiger Meinungsverschiedenheiten sind, d.h. solcher Kontroversen, zu deren Auflösung nicht auf die allein richtige Antwort auf eine Frage rekurriert werden kann (vgl. Hand 2008).

50 Die Frage, wie einfache Sachverhalte thematisiert werden sollten, erübrigt sich, denn in diesem Fall bedarf es keiner Erziehung bzw. ist Erziehung bereits an ihr Ende gekommen.

51 Ballauff, Th. (1993): Über die Unerlässlichkeit der Bildung. In: M Borelli (Hrsg.): Deutsche Gegenwartspädagogik. Band 1. Baltmannsweiler: Schneider, S. 1-19, hier: S. 4.

52 Ebd., S. 5. 